\title{
Inhibition of tumor growth and angiogenesis of tamoxifen-resistant breast cancer cells by ruxolitinib, a selective JAK2 inhibitor
}

\author{
JI WON KIM ${ }^{1}$, JAYA GAUTAM ${ }^{2}$, JI EUN KIM ${ }^{1}$, JUNG-AE KIM ${ }^{2}$ and KEON WOOK KANG ${ }^{1}$ \\ ${ }^{1}$ College of Pharmacy and Research Institute of Pharmaceutical Sciences, Seoul National University, Seoul 08826; \\ ${ }^{2}$ College of Pharmacy, Yeungnam University, Gyeongsan, Gyeongsangbuk-do 712-749, Republic of Korea
}

Received June 18, 2018; Accepted January 31, 2019

DOI: $10.3892 / \mathrm{ol} .2019 .10059$

\begin{abstract}
Tamoxifen (TAM) is the most widely used treatment for estrogen receptor-positive breast cancer patients. Unfortunately, the majority of these patients exhibit TAM resistance following treatment. We previously reported that proliferation and migration were greater in TAM-resistant MCF-7 (TAMR-MCF-7) cells than in parental MCF-7 cells. Janus kinases (JAKs) are cytosolic tyrosine kinases that transduce signals from plasma membrane cytokines and growth factor receptors. JAK2 selectively phosphorylates signal transducer and activator of transcription (STAT)-3, and the JAK2-STAT3 signaling pathway is known as a crucial signaling pathway for the regulation of cancer progression and metastasis. In the present study, basal phosphorylation of
\end{abstract}

Correspondence to: Professor Keon Wook Kang, College of Pharmacy and Research Institute of Pharmaceutical Sciences, Seoul National University, 1 Gwanak-ro, Gwanak-gu, Seoul 08826, Republic of Korea

E-mail: kwkang@snu.ac.kr

Professor Jung-Ae Kim, College of Pharmacy, Yeungnam University, 280 Daehak-ro, Gyeongsan, Gyeongsangbuk-do 712-749, Republic of Korea

E-mail: jakim@yu.ac.kr

Abbreviations: TAM, tamoxifen; CAM, chorioallantoic membrane; DAPI, 4'6-diamidino-2-phenylindole; DMEM, Dulbecco's modified Eagle's medium; EDTA, ethylenediamine tetraacetic acid; EGFR, epidermal growth factor; EMT, epithelial to mesenchymal transition; ER, estrogen receptor; FBS, fetal bovine serum; FGFR, fibroblast growth factor receptor; IGFR, insulin-like growth factor; IL-6, interleukin-6; JAK, Janus kinase; MMP2, matrix metalloproteinase-2; MMP9, matrix metalloproteinase-9; MPN, myeloproliferative neoplasm; PBS, phosphate-buffered saline; PDGFR, platelet-derived growth factor receptor; RT, room temperature; SDS, sodium dodecyl sulfate; STAT, signal transducer and activator of transcription; VEGF, vascular endothelial growth factor

Key words: ruxolitinib, JAK2, STAT3, tamoxifen-resistant breast cancer
STAT3 was revealed to be greater in TAMR-MCF-7 cells than in control MCF-7 cells. Ruxolitinib, a potent JAK2 inhibitor, was demonstrated to attenuate STAT3 phosphorylation and the proliferation of TAMR-MCF-7 cells. Ruxolitinib also suppressed the enhanced cell migration of TAMR-MCF-7 cells through the inhibition of epithelial mesenchymal transition. Vascular endothelial growth factor (VEGF), a representative target gene of the JAK2-STAT3 pathway, functions as a key regulator of invasion and angiogenesis. Ruxolitinib significantly inhibited VEGF mRNA expression and transcriptional activity. The present study also performed a chick embryo chorioallantoic membrane assay to assess tumor growth and angiogenesis in TAMR-MCF-7 cells. Ruxolitinib reduced tumor weight and the number of blood vessels produced by TAMR-MCF-7 cells in a concentration-dependent manner. These results indicated that JAK 2 could be a new therapeutic target for TAM-resistant breast cancer.

\section{Introduction}

Breast cancer is the most common malignancy among women and estrogen is an essential stimulant of estrogen receptor (ER)-positive breast cancer $(1,2)$. Tamoxifen (TAM), a non-steroidal anti-estrogen, is the most commonly used therapeutic or preventative agent for ER-positive breast cancer patients (3). However, acquired resistance to TAM is a critical problem with long-term TAM use, and the mechanism underlying this resistance remains unclear (4). We previously established an MCF-7-derived TAM-resistant cell line (TAMR-MCF-7 cells) by long-term incubation with 4-hydroxytamoxifen (5), and determined that TAMR-MCF-7 cells lost polarity and acquired migratory and invasive properties with higher expression of epithelial mesenchymal transition (EMT) marker proteins (6-8). This process is considered a prerequisite for tumor infiltration and metastasis (9). TAMR-MCF-7 cells have also been shown to exhibit enhanced vascular endothelial growth factor (VEGF) expression levels, leading to the promotion of angiogenesis (10).

Several receptors and transcription factors are actively involved in the regulation of cell proliferation and acquisition of migratory and invasive properties in diverse cancer 
cell types. Among these, the Janus kinase 2 (JAK2)-signal transducer and activator of transcription (STAT) 3 pathway transfers extracellular signals from cytokines and growth factor receptors, including the interleukin-6 receptor (IL-6R) and platelet-derived growth factor receptor (PDGFR), to its downstream target genes (11-13). Activation of the JAK-STAT pathway in cancer cells activates the transcription of several oncogenic genes, including c-myc, ccnd1, and VEGF (14-16). Several studies have indicated that the JAK2-STAT3 signaling pathway is a crucial activator of cell migration and cancer metastasis (17-19).

Ruxolitinib (Jakafi), a potent JAK1/2 inhibitor, was approved by the United States Food and Drug Administration (FDA) in 2011 for the treatment of patients with myeloproliferative neoplasia (MPN). Ruxolitinib directly inhibits JAK1 and JAK2 kinases, with $\mathrm{IC}_{50}$ values of 3.3 and $2.8 \mathrm{nM}$, respectively (20). Ruxolitinib reduced protein levels of phosphorylated JAK2 and subsequently inhibited protein expression of its downstream target genes, c-Myc, cyclin D1 and Bcl-2 (21). In preclinical studies, ruxolitinib was shown to inhibit the proliferation of JAK2 $2^{V 617 F}$-positive $\mathrm{Ba} / \mathrm{F} 3$ cells showing a constitutively active mutant form of JAK2, and to alleviate MPN symptoms in JAK2 $2^{V 617 F}$-transgenic mice (20). Moreover, involvement of JAK2 in cell proliferation was experimentally verified in various cancer cell lines, lung cancer cells (H661, H1975, H1563, ADOR, NSCLC1), breast cancer cells (MCF-7, MCF-7-HER18, SUM149, BT474, BT549, SKBR3) and glioblastoma cells (GBM6, GBM12) (21-23). However, the therapeutic effects of ruxolitinib on chemo-resistant breast cancer cells, and especially TAM-resistant cells, remain obscure. In the present study, we demonstrated for the first time that TAM-resistant MCF-7 cells showed higher sensitivity to ruxolitinib compared to parental MCF-7 cells, and the JAK inhibitor blocked the EMT process and VEGF production, consequently suppressing TAMR-MCF-7 cell migration and angiogenesis.

\section{Materials and methods}

Cell culture and reagents. MCF-7 (ER-positive human breast cancer cell line) cells were obtained from Korea Cell Line Bank (\#30022, 2005), and cultured at $37^{\circ} \mathrm{C}$ in Dulbecco's modified Eagle's medium (DMEM) containing $10 \%$ fetal bovine serum (FBS), $100 \mathrm{U} / \mathrm{ml}$ penicillin, and $100 \mu \mathrm{g} / \mathrm{ml}$ streptomycin. TAMR-MCF-7 cells were established as previously described $(5,24)$.

The antibodies used in the present study were as follow: phospho-STAT3 (p-STAT3) (Y705, cat. no. 9131S), STAT3 (cat. no. $4904 \mathrm{~S}$ ), Bcl-2 (cat. no. $2876 \mathrm{~S}$ ) and hypoxia inducible factor (HIF)-1 $\alpha$ (cat. no. 3716S) were purchased from Cell Signaling Technology (Beverly, MA, USA), c-Myc (cat. no. sc-40), c-Jun (cat. no. sc-1694), Cyclin B1 (cat. no. sc-245), Cyclin D1 (cat. no. sc-753), vimentin (cat. no. sc-32322), twist (cat. no. sc-81417) were obtained from Santa Cruz Biotechnology (Dallas, TX, USA), and glyceraldehyde 3-phosphate dehydrogenase (GAPDH, Calbiochem, Gibbstown, NJ, USA; cat. no. CB-1001), $\beta$-actin (Sigma-Aldrich, St. Louis, MO, USA; cat. no. a2228), E-cadherin (BD Biosciences, San Jose, CA, USA; cat. no. 610181), N-cadherin (BD Biosciences; cat. no. 610920), snail (Abcam, Cambridge, UK; cat. no. ab53519) were used. VEGF-luc plasmids were kindly donated from Dr. Lee (Chonnam National University, Gwangju, Korea).

Western blot analysis. Cells were lysed with lysis buffer

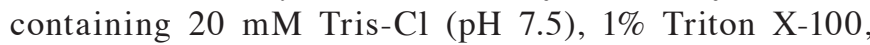
$137 \mathrm{mM}$ sodium chloride, $10 \%$ glycerol, 2 mM EDTA, $1 \mathrm{mM}$ sodium orthovanadate, $25 \mathrm{mM} \beta$-glycerophosphate, $2 \mathrm{mM}$ sodium pyrophosphate, $1 \mathrm{mM}$ phenylmethylsulfonylfluoride and $1 \mu \mathrm{g} / \mathrm{ml}$ leupeptin. The cell lysates were centrifuged at $13,000 \mathrm{x} g$ for $15 \mathrm{~min}$ to remove insoluble material, the supernatants were quantified using the Bradford method (25) and fractionated using polyacrylamide gel, and electrophoretically transferred to nitrocellulose membranes. Horseradish peroxidase (HRP)-conjugated anti-IgG antibodies were used as the secondary antibodies (cat. no. 7076S, 7074S, 1:5,000 dilution; Cell Signaling Technology). The nitrocellulose papers were developed using an ECL chemiluminescence system (Milipore, Billerica, MA, USA). For ECL chemiluminescence detection, LAS-3000 mini system (Fujifilm, Tokyo, Japan) was used.

Immunocytochemistry. MCF-7 and TAMR-MCF-7 cells were cultured overnight on coverslips. After treatment with $10 \mu \mathrm{M}$ ruxolitinib or vehicle, the cells were fixed with $4 \%$ paraformaldehyde and blocked with $1 \%$ bovine serum albumin at room temperature for $1 \mathrm{~h}$. The cells were incubated with Alexa Fluor 488-conjugated Phalloidin antibody (1:200 dilution, Thermo Fisher Scientific, Waltham, MA, USA) in $0.1 \%$ Tween-20-containing phosphate-buffered saline at $4^{\circ} \mathrm{C}$ overnight. Mouse monoclonal E-cadherin or $\mathrm{N}$-cadherin antibody (1:200 dilution, BD Biosciences) were also incubated in the same buffer at $4^{\circ} \mathrm{C}$ overnight. After twice washing with PBS, the coverslips were incubated with goat anti-mouse Alexa Fluor 568 IgG (1:2,000 dilution; Thermo Fisher Scientific, Inc., Waltham, MA, USA) at room temperature for $1 \mathrm{~h}$. Finally, the coverslips were washed thoroughly with PBS and then mounted with ProLong Gold Antifade reagent with 4',6-diamidino-2-phenylindole (DAPI; Invitrogen, Carlsbad, CA, USA). Images were obtained using CELENAS Digital Imaging System (Logos Biosystems, Anyang, South Korea).

Cell proliferation assay. MCF-7 and TAMR-MCF-7 cells were seeded in 96-well plate ( $3 \times 10^{3}$ cells/well) and cultured for $96 \mathrm{~h}$ in the presence or absence of ruxolitinib (0.1-10 $\mu \mathrm{M})$. The kinetics of viable cell numbers were counted using IncuCyte ZOOM live-cell analysis system (Essen BioScience, Ann Arbor, MI, USA) and monitored using the IncuCyte label-free cell monolayer confluence algorithm. IncuCyte provides the ability to acquire phase-contrast images and an integrated confluence metric as a surrogate for cell number.

Transwell migration assay. TAMR-MCF-7 cells were seeded in the upper chamber of the transwell plate (Essen BioScience) and the lower side of the upper chamber was covered with type I collagen (Sigma-Aldrich). The lower chamber was filled with $200 \mu \mathrm{l} \mathrm{FBS-containing} \mathrm{culture}$ media. The cells were incubated at $5 \% \mathrm{CO}_{2}$ for $18 \mathrm{~h}$. The migrated cell numbers were counted using IncuCyte ZOOM live-cell analysis system. 
Semi-quantitative polymerase chain reaction ( $s q P C R)$. Total RNA was isolated from MCF-7 and TAMR-MCF-7 cells using Trizol ${ }^{\circledR}$ reagent (Invitrogen), and cDNA was synthesized by reverse transcriptase using an oligo (dT) primer. PCR was performed using the selective primers for human VEGF (sense: 5'-GCTACTGCCATCCAATCGAG-3', antisense: 5'-TGCATT CACATTTGTTGTGC-3'), human GAPDH (sense: 5'-AAG GCTGAGAACGGGAAG-3', antisense: 5'-GCCCCACTT GATTTTGGA-3') as the housekeeping gene. sqPCR was performed for 40 cycles (denaturation at $98^{\circ} \mathrm{C}$ for $30 \mathrm{sec}$, annealing at $60^{\circ} \mathrm{C}$ for $30 \mathrm{sec}$, and then a final extension at $60^{\circ} \mathrm{C}$ for $\left.5 \mathrm{~min}\right)$. After PCR, $6 \mu \mathrm{l}$ samples of the products were subjected to $2.0 \%$ agarose gel electrophoresis and stained with ethidium bromide. Densitometric analyses were performed using Quantity One 1-D Analysis Software version 4.6.2 (Bio-Rad Laboratories, Inc., Hercules, CA, USA).

Reporter gene assay. MCF-7 and TAMR-MCF-7 cells $\left(1 \times 10^{5}\right.$ cells/well) were seeded in 48 -well plates, then transfected with luciferase reporter plasmids containing VEGF-luc reporter plasmid containing the luciferase structural gene and the human VEGF promoter and pRL-SV plasmids (Renilla luciferase expression for normalization). The transfected cells were exposed to compound for $24 \mathrm{~h}$, and the promoter activity was measured using a dual-luciferase reporter assay system (Promega, Madison, WI, USA). The firefly and Renilla luciferase activities were measured using a luminometer (LB941, Berthold Technologies, Bad Wildbad, Germany).

Chick chorioallantoic membrane (CAM) assay. Fertilized eggs were purchased from Siprigol Poultry Farm (Gyeongsan, South Korea) and the CAM was prepared as described previously (26-28). The surfaces of 10-day-old post-fertilization chick eggs were sterilized and the CAM was exposed by cutting a window $\left(1 \mathrm{~cm}^{2}\right)$ on one side of the egg using the false air sac technique. TAMR-MCF-7 cells were placed on the exposed CAM and the windows were sealed with transparent tape. The eggs were then incubated in a humidified incubator at $37^{\circ} \mathrm{C}$. The CAMs were examined at 3 days after inoculation using a SV6 stereomicroscope (Carl Zeiss, Hamburg, Germany) at $50 \mathrm{x}$ magnification. The CAM experiments were terminated at 13th embryonic day. After extraction of chorioallantoic membrane, chick embryos should be dead due to the excess hemorrhage. Then the dead cadaver was disposed in accordance with the biological waste treatment procedures. Digital images of CAM sections were collected using a 3-charge coupled device color video camera system (Toshiba, Tokyo, Japan). The number of vessel branch points contained in a circular region was counted. The experimental studies were approved by the Institutional Animal Care and Use Committee of Yeungnam University (Gyeongsangbuk, Korea).

Statistical analysis. Statistical analysis was performed using Sigma plot 12.0 (Systat Software, Inc., San Jose, CA, USA) with one-way analysis of variance and Tukey's post hoc multiple comparisons test to determine the differences. $\mathrm{P}<0.05$ was considered to indicate a statistically significant difference.

\section{Results}

Ruxolitinib inhibits STAT3 activation in TAMR-MCF-7 cells. We compared JAK2 activity between MCF-7 and TAMR-MCF-7 cells by determining the extent of Tyr705 STAT3 phosphorylation. Immunoblot results showed that STAT3 phosphorylation was much higher in TAMR-MCF-7 cells than in control MCF-7 cells (Fig. 1A). We then assessed whether ruxolitinib disturbed the JAK2-STAT3 signaling pathway in both breast cancer cell types, and found that p-STAT3 levels decreased with ruxolitinib treatment (0.1-10 $\mu \mathrm{M})$ in a concentration-dependent manner in both MCF-7 and TAMR-MCF-7 cells (Fig. 1B). Many studies have reported that STAT3 activation is required for the transactivation of several oncoproteins and survival factors, including c-Myc, c-Jun, Cyclin B1, Cyclin D1, Bcl-2, and HIF-1 $\alpha$ (29,30). In comparison to protein levels of MCF-7 cells, those of c-Myc, c-Jun, Cyclin B1, Cyclin D1, Bcl-2, and HIF-1 $\alpha$ were upregulated in TAMR-MCF-7 cells, and this enhanced expression was markedly inhibited by ruxolitinib (0.1-10 $\mu \mathrm{M}$, Fig. 1C). These results demonstrate that ruxolitinib attenuates activation of the JAK2-STAT3 pathway in TAMR-MCF-7 cells.

Ruxolitinib inhibits TAMR-MCF-7 cell proliferation. To determine the effect of ruxolitinib on breast cancer cell proliferation, real-time, live-cell monitoring and analysis were performed using IncuCyte ZOOM. Ruxolitinib significantly inhibited the growth of MCF-7 cells at a concentration of $10 \mu \mathrm{M}$ (Fig. 2A). However, TAMR-MCF-7 cells showed enhanced sensitivity to ruxolitinib (Fig. 2B). When ruxolitinib at the concentration of 1 or $3 \mu \mathrm{M}$ was exposed to TAMR-MCF7 cells, the inhibiting effect of cell growth was slight but significant. Also, this inhibitory effect was not observed in MCF-7 cells treated with ruxolitinib at the same concentration ranges. These data suggest that TAMR-MCF-7 cell proliferation is partially dependent on the JAK2-STAT3 pathway and that ruxolitinib preferentially suppresses TAM-resistant breast cancer cell proliferation.

Ruxolitinib inhibits cell migration and EMT progression in TAMR-MCF-7 cells. Because the JAK2-STAT3 pathway plays an important role in cancer cell migration and invasion, we performed a transwell migration assay to assess TAMR-MCF-7 cell migration. We previously reported that TAMR-MCF-7 cells possess greater in vitro migratory ability than MCF-7 cells (8). TAMR-MCF-7 cell migration was significantly suppressed under treatment with $10 \mu \mathrm{M}$ ruxolitinib (Fig. 3A). Because TAMR-MCF-7 cells acquire the migratory phenotype via EMT progression (7), we then examined whether JAK2-STAT3 inhibition by ruxolitinib affects the expression of EMT markers in TAMR-MCF-7 cells. Representative biochemical markers of EMT include loss of the epithelial adherence protein E-cadherin and upregulation of the mesenchymal protein $\mathrm{N}$-cadherin (9). Immunocytochemistry for E-cadherin, N-cadherin, and phalloidin in MCF-7 and TAMR-MCF-7 cells showed that E-cadherin downregulation and $\mathrm{N}$-cadherin upregulation in TAMR-MCF-7 cells were partially reversed by treatment with $10 \mu \mathrm{M}$ ruxolitinib (Fig. 3B). Western blot analyses confirmed that higher expression of mesenchymal marker proteins, such as $\mathrm{N}$-cadherin, vimentin, snail, or twist, 

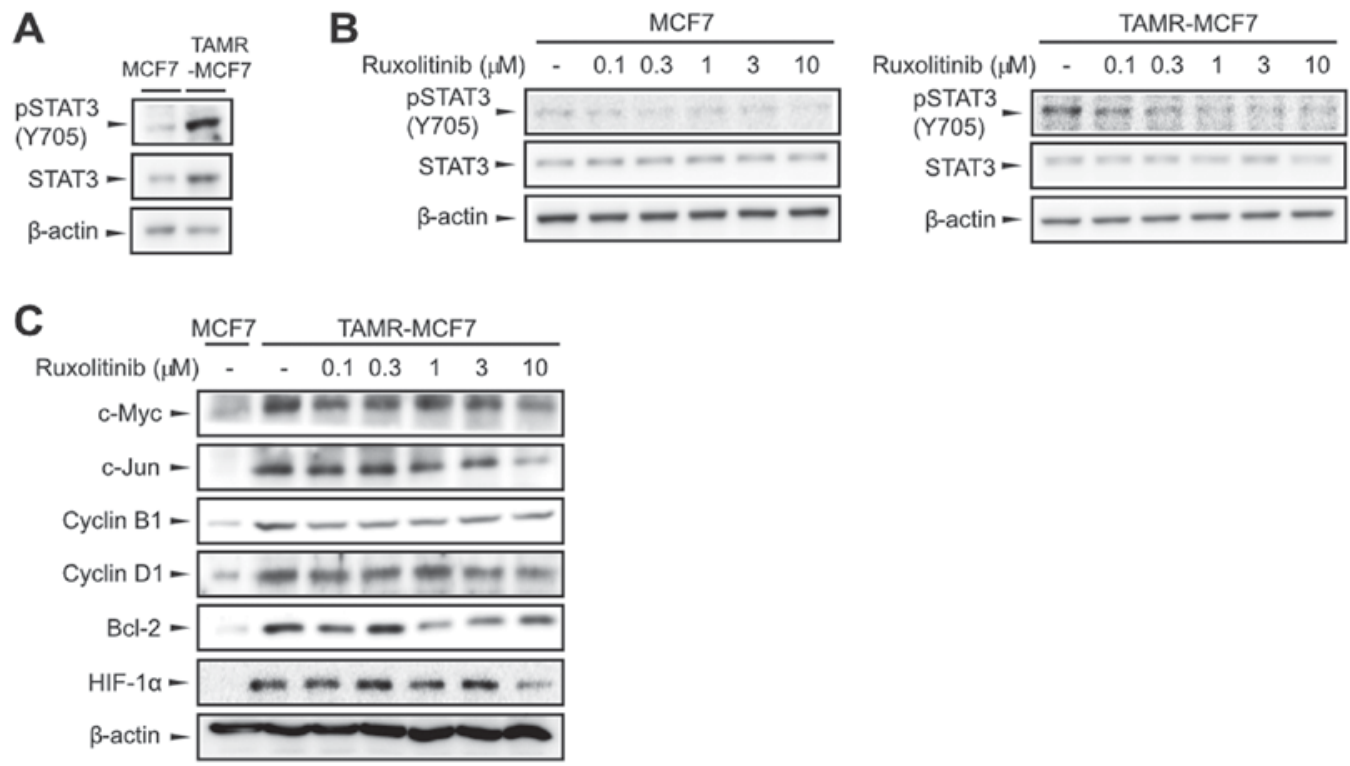

Figure 1. Inhibition of the STAT3 signaling pathway by ruxolitinib in TAMR-MCF-7 cells. (A) Expression of pSTAT3 and STAT3 in MCF-7 and TAMR-MCF-7 cells. $\beta$-actin levels were utilized as the loading controls. (B) Inhibition of STAT3 phosphorylation by ruxolitinib. MCF-7 and TAMR-MCF-7 cells were treated with vehicle or ruxolitinib $(0.1-10 \mu \mathrm{M})$ for $24 \mathrm{~h}$. (C) Effects of ruxolitinib on the expression of downstream target genes of the JAK-STAT pathway. The protein levels of c-Myc, c-Jun, Cyclin B1, Cyclin D1, Bcl-2 and HIF-1 $\alpha$ were determined in MCF-7 and TAMR-MCF-7 cells $24 \mathrm{~h}$ following ruxolitinib treatment $(0.1-10 \mu \mathrm{M})$. All of the experiments were repeated at least three times. JAK, Janus kinase; STAT, signal transducer and activator of transcription; pSTAT, phosphorylated STAT; HIF-1 $\alpha$, hypoxia inducible factor-1 $\alpha$; Bcl-2, B-cell lymphoma 2; TAMR, tamoxifen resistant.
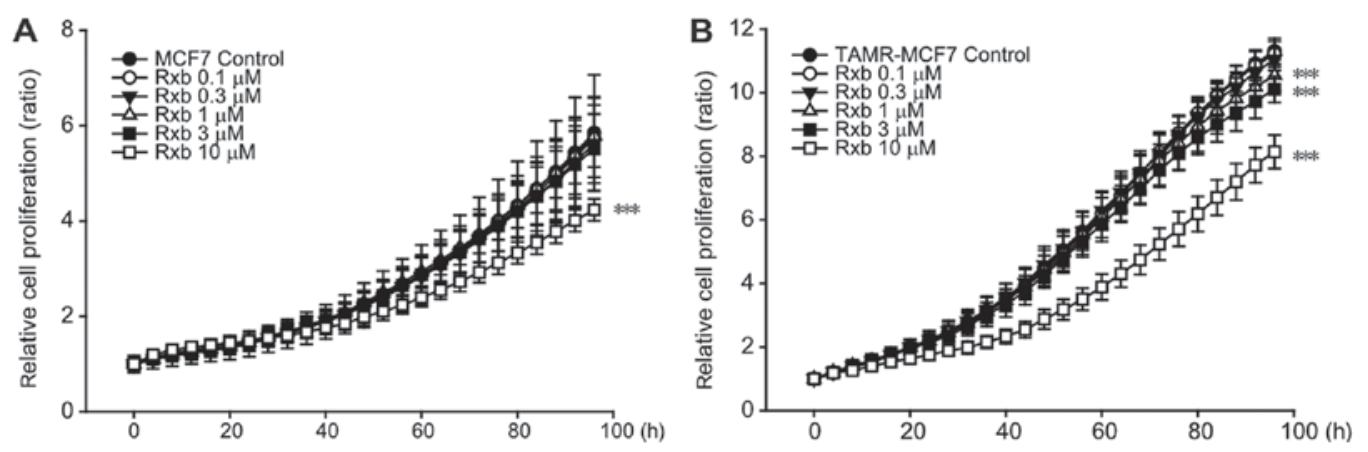

Figure 2. Anti-proliferation effects of ruxolitinib in breast cancer cells. (A and B) Effects of ruxolitinib on the cell proliferation of (A) MCF-7 and (B) TAMR-MCF-7 cells. MCF-7 and TAMR-MCF-7 cells were cultured in 96-well plate and cell proliferation was determined by IncuCyte ZOOM live-cell analysis system for $96 \mathrm{~h}$. Data represent means \pm standard deviation $(\mathrm{n}=6) .{ }^{* * * *} \mathrm{P}<0.005$ vs. vehicle-treated control. Rxb, ruxolitinib; TAMR, tamoxifen resistant.

was suppressed in TAMR-MCF-7 cells by ruxolitinib in a concentration-dependent manner (Fig. 3C). Although E-Cadherin was slightly detected in TAMR-MCF-7 cells by immunocytochemistry, E-cadherin was not detectable by immunoblottings in TAMR-MCF7 cells (Fig. 3C) (8). It may result from the difference in detection sensitivity between immunoblotting and immunocytochemistry. These results demonstrate that ruxolitinib may inhibit cell migration in TAMR-MCF-7 cells, presumably by blocking EMT.

Ruxolitinib inhibits angiogenesis and tumor growth. A clinical feature of TAM resistance in human breast cancer is an increase in microvessel counts (31). We previously reported that angiogenic potential was enhanced in TAMR-MCF-7 cells by VEGF upregulation (10). In the current study, high basal VEGF mRNA expression was observed in TAMR-MCF-7 cells, but not in MCF-7 cells (Fig. 4A). Consistent with the inhibitory effects of ruxolitinib on cell migration and EMT progression of TAMR-MCF-7 cells, ruxolitinib $(0.1-10 \mu \mathrm{M})$ reduced VEGF mRNA levels in TAMR-MCF-7 cells (Fig. 4A). Moreover, a VEGF-luc reporter gene assay further revealed that VEGF promoter binding activity was significantly diminished by treatment with 0.3-1 $\mu \mathrm{M}$ ruxolitinib (Fig. 4B). As ruxolitinib above $3 \mu \mathrm{M}$ causes a significant cell death in the reporter gene analysis condition because of the lipid carrier-based membrane damage (32), MCF-7 and TAMR-MCF-7 cells were exposed to $0.3-1 \mu \mathrm{M}$ ruxolitinib. These results suggest that the overexpression of VEGF gene transcription in TAMR-MCF-7 cells was partly a result of JAK2-STAT3 pathway activation. We then performed chorioallantoic membrane (CAM) assays by placing TAMR-MCF-7 cells on CAMs to verify the effect of ruxolitinib on angiogenesis. Numbers of vessel branch points in TAMR-MCF-7 cells were significantly decreased by ruxolitinib in a concentration-dependent manner (Fig. 4C).

To estimate the inhibitory effect of ruxolitinib on tumor growth, we analyzed the tumor weight of TAMR-MCF-7 cells 
A
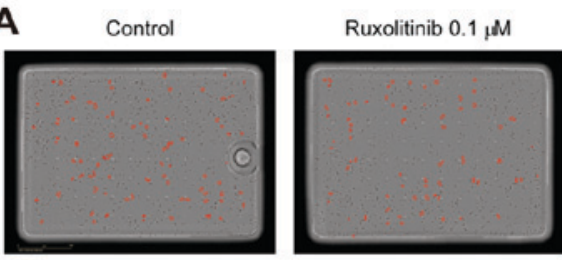

B
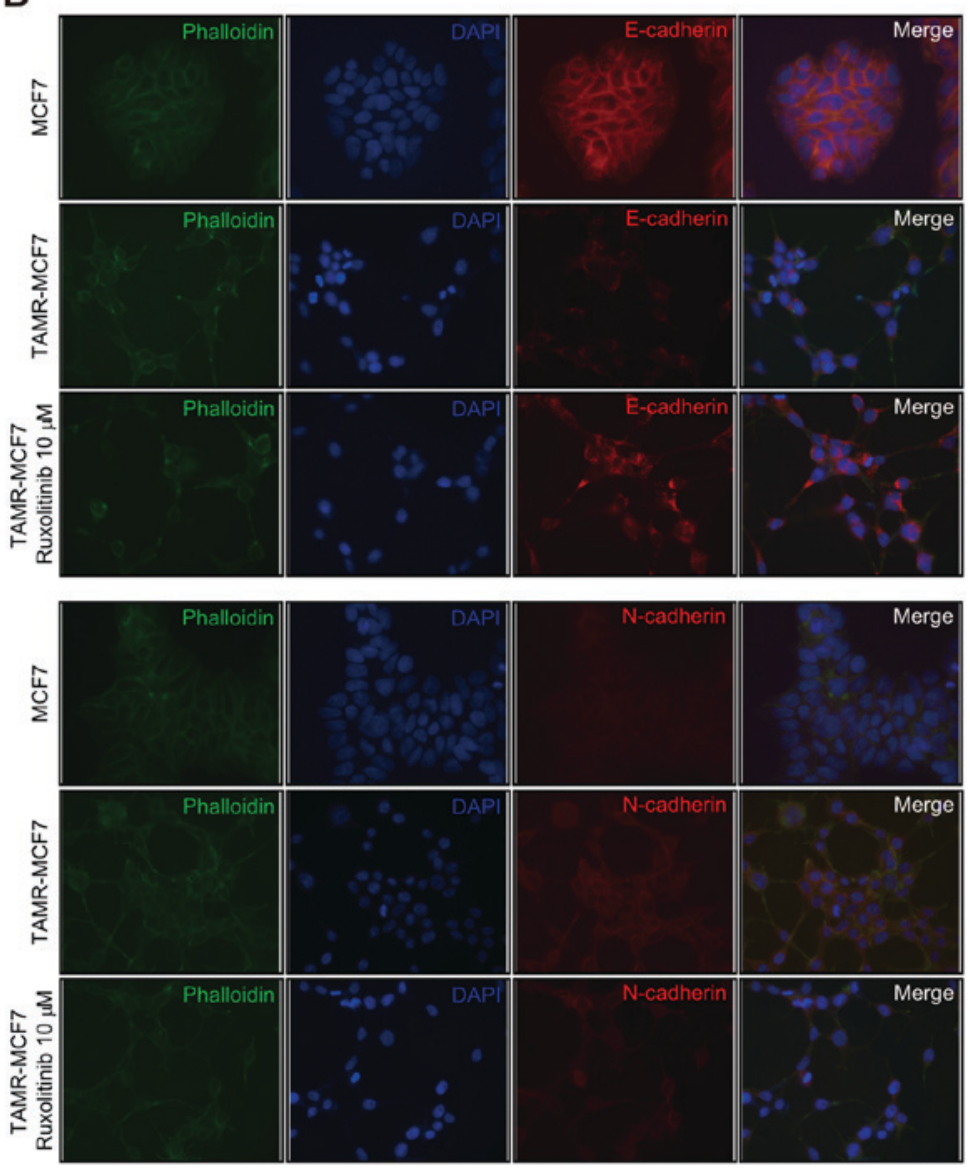

Ruxolitinib $1, \mathrm{M}$
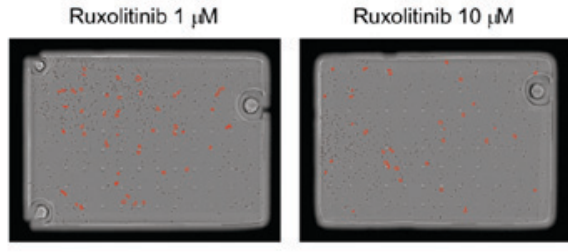

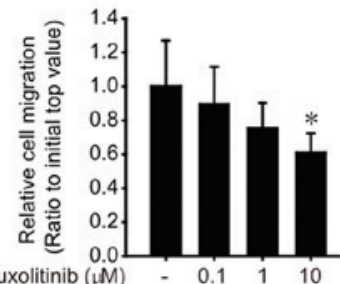

C

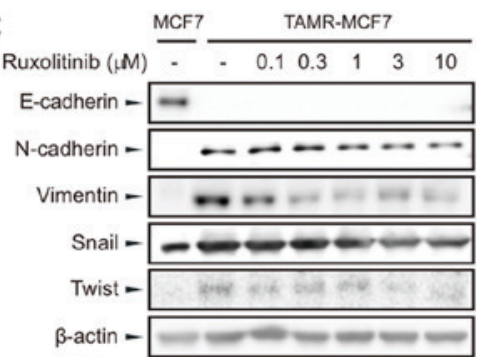

Figure 3. Effects of ruxolitinib on cell migration and EMT phenotype changes in TAMR-MCF-7 cells. (A) Effect of ruxolitinib on TAMR-MCF-7 cell migration. Transwell migration assays were performed in TAMR-MCF-7 cells $18 \mathrm{~h}$ post-treatment with ruxolitinib (0.1-10 $\mu \mathrm{M})$. Representative pictures of migrated cells (red circles) were shown (left panel). The relative cell numbers of migrated cells were counted (right panel). Data are presented as the mean \pm standard deviation ( $\mathrm{n}=4)$. ${ }^{*} \mathrm{P}<0.05$ vs. vehicle-treated control. (B) Immunofluorescence staining of EMT markers in MCF-7 and TAMR-MCF-7 cells. TAMR-MCF-7 cells cultured on coverslips were incubated with or without $10 \mu \mathrm{M}$ ruxolitinib for $24 \mathrm{~h}$, and then the fixed cells were stained with specific antibodies against phalloidin, E-cadherin or N-cadherin. Representative images were captured using a CELENAS Digital Imaging System. Magnification, x40. (C) Expression of EMT markers in TAMR-MCF-7 cells. EMT phenotype markers were determined following the exposure of TAMR-MCF-7 cells to 0.1-10 $\mu$ M ruxolitinib for 24 h. EMT, epithelial mesenchymal transition; TAMR, tamoxifen resistant.

on CAMs. Implanted tumor growth was strongly suppressed by ruxolitinib (0.3-3 $\mu \mathrm{M})$ (Fig. 4D), which implies that JAK2-STAT3 signaling is also critical to in vivo tumor growth in TAM-resistant breast cancer.

\section{Discussion}

As a selective ER modulator, TAM is the most widely used treatment for ER-positive breast cancer in both premenopausal and postmenopausal patients (3). Approximately $70 \%$ of breast cancer patients express ER; therefore, ER is the major target for luminal breast cancer therapy (33). Nonetheless, intrinsic or acquired resistance to hormonal therapy is the main challenge to clinicians, with $50 \%$ of advanced metastatic breast cancer patients developing resistance to TAM (34). Several studies have suggested potential mechanisms underlying the acquisition of TAM resistance, and therapeutic targets that may cause drug sensitivity in TAM-resistant breast cancer. Overexpression or formation of ER variants, coregulator switching, abnormal microRNA expression, and genetic polymorphism have been suggested as possible mechanisms for TAM resistance $(34,35)$. In addition, the dysregulation of intracellular signaling has been seen during the acquisition of TAM resistance in several contexts, including growth factor receptors [epidermal growth factor receptor (EGFR/HER2), fibroblast growth factor receptor, type-I insulin-like growth factor receptor (IGFIR)), 
A

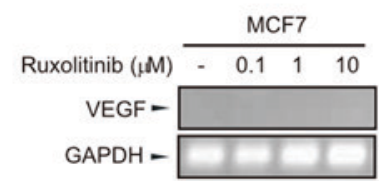

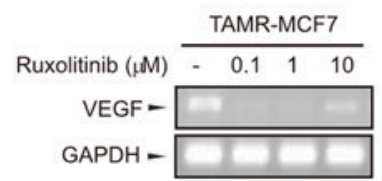

B

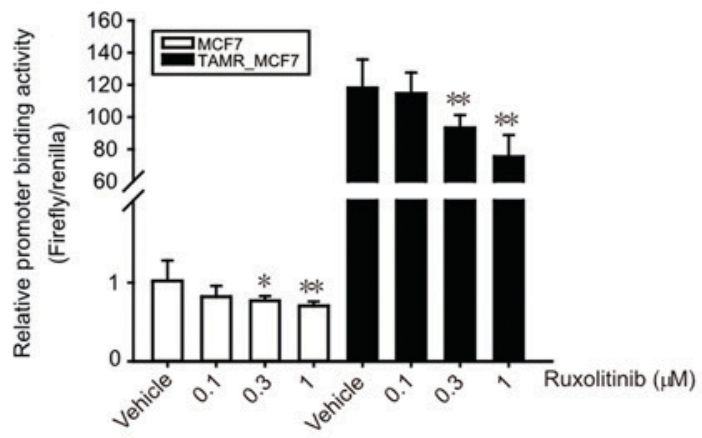

\section{C $\quad$ TAMR-MCF7}

Ruxolitinib
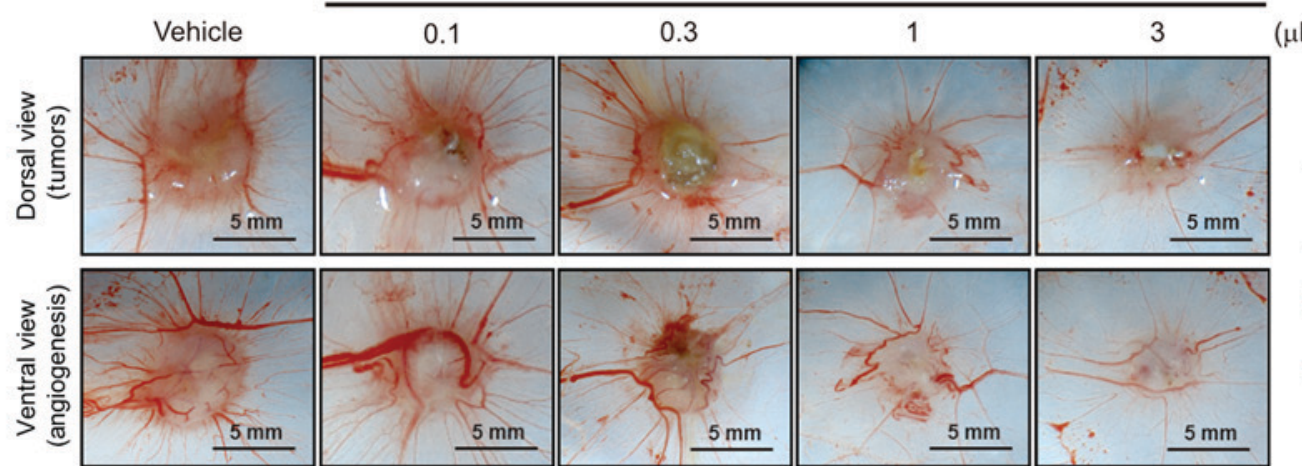

$(\mu \mathrm{M})$

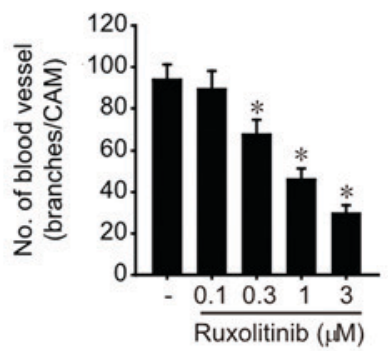

\section{D}
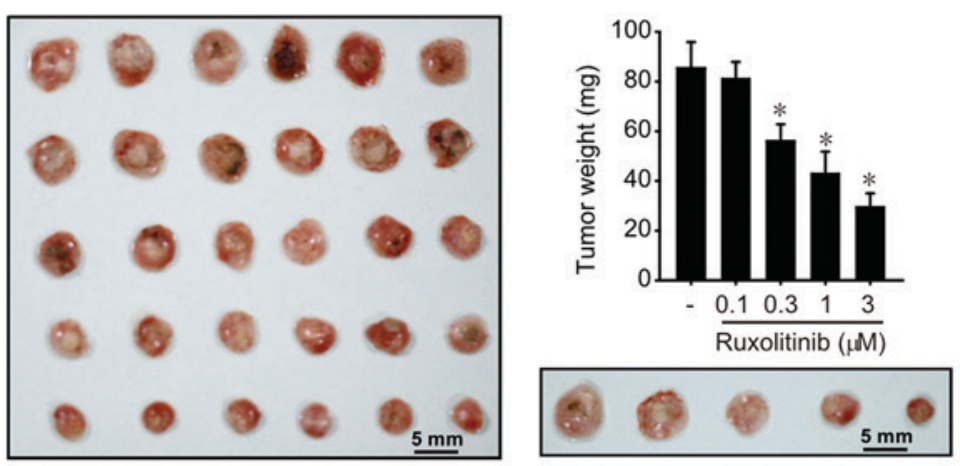

Figure 4. Effect of ruxolitinib on VEGF-induced angiogenesis in breast cancer cells. (A) mRNA levels of VEGF. sqPCR was performed in MCF-7 and TAMR-MCF-7 cells. The mRNA expression levels were normalized to GAPDH mRNA. (B) Concentration-dependent effects of ruxolitinib on VEGF gene transcription in MCF-7 and TAMR-MCF-7 cells. MCF-7 and TAMR-MCF-7 cells were transfected with VEGF-luc and pRL-SV plasmids and the cells were incubated with or without 0.1-1 $\mu \mathrm{M}$ ruxolitinib for $24 \mathrm{~h}$. (C and D) Inhibitory effects of ruxolitinib on TAMR-MCF-7-induced (C) angiogenesis and (D) tumor growth (scale bars, $5 \mathrm{~mm}$ ). Representative images of angiogenesis originated from TAMR-MCF-7. The control CAM of a 10-day-old chick embryo was exposed to PBS. The additional embryos were implanted with TAMR-MCF-7 cells ( $2 \times 10^{6}$ cells/CAM) and exposed to $0.1-3 \mu$ M ruxolitinib. The quantitation of new branches formed from existing blood vessels was performed 3 days following cancer cell implantation. Data are presented as the mean \pm standard deviation from 6 different samples. ${ }^{*} \mathrm{P}<0.05$ and ${ }^{* * *} \mathrm{P}<0.01$ vs. vehicle-treated control. CAM, chorioallantoic membrane; VEGF, vascular endothelial growth factor; sqPCR, semi-quantitative polymerase chain reaction; TAMR, tamoxifen resistant.

phosphoinositide 3-kinase (PI3K)-phosphatase and tensin homolog (PTEN)/AKT/mammalian target of rapamycin (mTOR), and nuclear factor- $\mathrm{\kappa B}(\mathrm{NF}-\mathrm{\kappa B})$ (36). In particular, the activation of EGFR/HER2 or IGFR is a key molecular pathway implicated in TAM resistance (4). However, clinical trials combining anti-estrogens with EGFR inhibitors have provided minimal, or no, clinical benefit to patients over anti-estrogens alone $(37,38)$. Because hormone resistance to breast cancer typically appears to be caused by diverse factors, identifying a new therapeutic target for the prevention of cancer relapse under continued hormone therapy remains problematic.

Activation of the JAK-STAT pathway has been considered an essential downstream signaling response to interferon (IFN)- $\alpha$, IFN- $\gamma$, and IL-6 (39). Because the JAK-STAT pathway is also involved in the proliferation, differentiation, migration, and apoptosis of cancer cells (40-42), diverse inhibitors have been developed to target the JAK family (43-46). Among these, tofacitinib (Xeljanz) and ruxolitinib have been approved for the treatment of rheumatoid arthritis, myelofibrosis, and polycythemia vera (PCV), and are now in clinical trials for novel therapeutic applications, such as colitis and various solid tumors (pancreatic cancer, breast cancer, and non-small-cell lung cancer (NSCLC) (45). It has been reported that ruxolitinib suppresses Hodgkin's lymphoma (HL) and primary mediastinal B-cell lymphoma (PMBL) growth in vitro, and increases programmed cell death against lymphoma cells (47). Ruxolitinib has also been shown to significantly inhibit tumor growth and 
improve survival in HL and PMBL xenograft mice (47). In the current study, we showed for the first time that STAT3 activity was highly increased in TAMR-MCF-7 cells, and that ruxolitinib inhibited cell proliferation and tumor growth in TAM-resistant breast cancer cells, though the inhibition intensity is marginal. We believe that the functional role of JAK2, which can be inhibited by ruxolitinib, is not mainly involved in the growth of TAMR-MCF7 cells. Lim et al (21) recently demonstrated that ruxolitinib and calcitriol possessed synergistic anticancer effect in MCF-7 cells. Although ruxolitinib showed marginal inhibition rate in cell growth of MCF-7 cells, synergistic inhibition of cell proliferation was observed by ruxolitinib/calcitriol combination. Based on the finding, we believe that ruxolitinib could be used as a regimen for combination therapy in TAM-resistant breast cancer patients. In fact, JAK-STAT has been reported to be constitutively active in a triple-negative breast cancer (TNBC) cell line, MDA-MB-cells, and TNBC tumor tissues $(12,48-50)$, and an oral JAK inhibitor suppresses tumor growth and metastasis of TNBC cells in vitro and in vivo (51). In a terminated clinical study, single administration of ruxolitinib showed no objective responses in a treatment-refractory TNBC patient population (52). The authors indicated that the discrepancy between clinical outcomes and on-target activity may be due to intratumoral heterogeneity within individual TNBC biopsies (50). Considering our cell-based results, tamoxifen-resistant breast cancer patients may be able to enroll in new clinical studies using ruxolitinib.

Increased metastasis and angiogenesis are the representative clinical characteristics of TAM-resistant breast cancer (31). We and other groups have shown that EMT contributes to the enhanced migration ability of TAM-resistant breast cancer cells, which may be required for higher rates of metastasis (7-9). During EMT progression, morphological changes to highly motile mesenchymal cancer cells are associated with several molecular characteristics, including increased levels of $\mathrm{N}$-cadherin and vimentin, reduced levels of E-cadherin and claudins, and the release of matrix remodeling enzymes such as matrix metalloproteinases (52). Changes in the expression patterns of these proteins have been shown to result from induction of EMT-activating transcription factors, such as the snail family of zinc finger transcription factors, basic helix-loop-helix factors, and twist (53). In the current study, protein expression of mesenchymal phenotype markers and transcription factors upregulated in TAMR-MCF-7 cells was diminished by ruxolitinib treatment. Consistent with our results, JAK1/2 inhibition by ruxolitinib suppressed STAT3-mediated expression of ZEB1 and snail as well as the emergence of a mesenchymal phenotype in hepatocellular carcinoma (54). It has been also reported that the JAK-STAT pathway is involved in VEGF gene transcription $(55,56)$. VEGF mRNA expression and gene transcription were highly upregulated in TAMR-MCF-7 cells, but were suppressed by ruxolitinib. Consistent with sqPCR results, a reporter gene assay using the VEGF-luc promoter showed that ruxolitinib inhibited the activation of VEGF gene transcription. It has been reported that protein expression and secretion of VEGF mainly depend on the transcriptional activation of the VEGF gene (10). Considering our findings that ruxolitinib inhibits both the VEGF-luc reporter activity and VEGF mRNA expression, VEGF protein levels may be down-regulated by ruxolitinib in TAMR-MCF-7 cells. The fact that we did not quantify the amount of secreted VEGF would be a limitation of our study. CAM assay results clearly demonstrated that ruxolitinib can suppress TAMR-MCF7-induced angiogenesis, where this effect was mediated by VEGF. In conclusion, ruxolitinib, a potent JAK-STAT pathway inhibitor, blocked the EMT process and VEGF production, consequently suppressing TAMR-MCF-7 cell migration and angiogenesis. These results hold great promise in improving the clinical outcomes of TAM-resistant breast cancer patients.

\section{Acknowledgements}

Not applicable.

\section{Funding}

The present study was supported by the National Research Foundation of Korea (NRF) grant (grant nos. NRF-2018R1A2B2003590 and 2017R1A4A1015860).

\section{Availability of data and materials}

The dataset generated and/or analysed during this study are available from the corresponding author on reasonable request.

\section{Author's contributions}

KWK and JAK designed the research. JWK, JG and JEK performed the research. JWK and JEK contributed to the western blot analysis, cell culture, sqPCR, luciferase assay and immunocytochemistry assay. JG contributed to the CAM assay and data analysis.

\section{Ethics approval and consent to participate}

The experimental studies were approved by the Institutional Animal Care and Use Committee of Yeungnam University (Gyeongsangbuk, Korea).

\section{Patient consent for publication}

Not applicable.

\section{Competing interests}

The authors declare that they have no competing interests.

\section{References}

1. Mueller SO, Clark JA, Myers PH and Korach KS: Mammary gland development in adult mice requires epithelial and stromal estrogen receptor alpha. Endocrinology 143: 2357-2365, 2002.

2. Petrangeli E, Lubrano C, Ortolani F, Ravenna L, Vacca A, Sciacchitano S, Frati L and Gulino A: Estrogen receptors: New perspectives in breast cancer management. J Steroid Biochem Mol Biol 49: 327-331, 1994.

3. Rose C, Thorpe SM, Andersen KW, Pedersen BV, Mouridsen HT, Blichert-Toft $\mathrm{M}$ and Rasmussen BB: Beneficial effect of adjuvant tamoxifen therapy in primary breast cancer patients with high oestrogen receptor values. Lancet 1: 16-19, 1985. 
4. Ali S and Coombes RC: Endocrine-responsive breast cancer and strategies for combating resistance. Nat Rev Cancer 2: 101-112, 2002.

5. Choi HK, Yang JW, Roh SH, Han CY and Kang KW: Induction of multidrug resistance associated protein 2 in tamoxifen-resistant breast cancer cells. Endocr Relat Cancer 14: 293-303, 2007.

6. Clarke R, Thompson EW, Leonessa F, Lippman J, McGarvey M, Frandsen TL and Brünner N: Hormone resistance, invasiveness and metastatic potential in breast cancer. Breast Cancer Res Treat 24: 227-239, 1993

7. Kim MR, Choi HK, Cho KB, Kim HS and Kang KW: Involvement of Pin1 induction in epithelial-mesenchymal transition of tamoxifen-resistant breast cancer cells. Cancer Sci 100 $1834-1841,2009$

8. Bui QT, Im JH, Jeong SB, Kim YM, Lim SC, Kim B and Kang KW: Essential role of Notch4/STAT3 signaling in epithelial-mesenchymal transition of tamoxifen-resistant human breast cancer. Cancer Lett 390: 115-125, 2017.

9. Christiansen JJ and Rajasekaran AK: Reassessing epithelial to mesenchymal transition as a prerequisite for carcinoma invasion and metastasis. Cancer Res 66: 8319-8326, 2006.

10. Kim MR, Choi HS, Yang JW, Park BC, Kim JA and Kang KW: Enhancement of vascular endothelial growth factor-mediated angiogenesis in tamoxifen-resistant breast cancer cells: Role of Pin1 overexpression. Mol Cancer Ther 8: 2163-2171, 2009.

11. Bollrath J, Phesse TJ, von Burstin VA, Putoczki T, Bennecke M, Bateman T, Nebelsiek T, Lundgren-May T, Canli O, Schwitalla S, et al: gp130-mediated Stat3 activation in enterocytes regulates cell survival and cell-cycle progression during colitis-associated tumorigenesis. Cancer Cell 15: 91-102, 2009.

12. Berishaj M, Gao SP, Ahmed S, Leslie K, Al-Ahmadie H, Gerald WL, Bornmann W and Bromberg JF: Stat3 is tyrosine-phosphorylated through the interleukin-6/glycoprotein 130/Janus kinase pathway in breast cancer. Breast Cancer Res 9: R32, 2007

13. Vignais ML, Sadowski HB, Watling D, Rogers NC and Gilman M: Platelet-derived growth factor induces phosphorylation of multiple JAK family kinases and STAT proteins. Mol Cell Biol 16: 1759-1769, 1996.

14. Vainchenker W and Constantinescu SN: JAK/STAT signaling in hematological malignancies. Oncogene 32: 2601-2613, 2013.

15. Yu H and Jove R: The STATs of cancer-new molecular targets come of age. Nat Rev Cancer 4: 97-105, 2004.

16. Furqan M, Mukhi N, Lee B and Liu D: Dysregulation of JAK-STAT pathway in hematological malignancies and JAK inhibitors for clinical application. Biomark Res 1: 5, 2013

17. Liu K, Gao H, Wang Q, Wang L, Zhang B, Han Z, Chen X, Han M and Gao M: Hispidulin suppresses cell growth and metastasis by targeting PIM1 through JAK2/STAT3 signaling in colorectal cancer. Cancer Sci 109: 1369-1381, 2018.

18. Wu X, Tao P, Zhou Q, Li J, Yu Z, Wang X, Li J, Li C, Yan M, Zhu Z, et al: IL-6 secreted by cancer-associated fibroblasts promotes epithelial-mesenchymal transition and metastasis of gastric cancer via JAK2/STAT3 signaling pathway. Oncotarget 8 : 20741-20750, 2017.

19. Wei Z, Jiang X, Qiao H, Zhai B, Zhang L, Zhang Q, Wu Y, Jiang H and Sun X: STAT3 interacts with Skp2/p27/p21 pathway to regulate the motility and invasion of gastric cancer cells. Cel Signal 25: 931-938, 2013.

20. Quintás-Cardama A, Vaddi K, Liu P, Manshouri T, Li J Scherle PA, Caulder E, Wen X, Li Y, Waeltz P, et al: Preclinical characterization of the selective JAK1/2 inhibitor INCB018424: Therapeutic implications for the treatment of myeloproliferative neoplasms. Blood 115: 3109-3117, 2010.

21. Lim ST, Jeon YW, Gwak H, Kim SY and Suh YJ: Synergistic anticancer effects of ruxolitinib and calcitriol in estrogen receptor-positive, human epidermal growth factor receptor 2-positive breast cancer cells. Mol Med Rep 17: 5581-5588, 2018.

22. Tavallai M, Booth L, Roberts JL, McGuire WP, Poklepovic A and Dent P: Ruxolitinib synergizes with DMF to kill via BIM+BAD-induced mitochondrial dysfunction and via reduced SOD2/TRX expression and ROS. Oncotarget 7: 17290-17300, 2016.

23. Tavallai M, Booth L, Roberts JL, Poklepovic A and Dent P. Rationally repurposing ruxolitinib (Jakafi (囚)) as a solid tumor therapeutic. Front Oncol 6: 142, 2016.
24. Knowlden JM, Hutcheson IR, Jones HE, Madden T, Gee JM, Harper ME, Barrow D, Wakeling AE and Nicholson RI: Elevated levels of epidermal growth factor receptor/c-erbB2 heterodimers mediate an autocrine growth regulatory pathway in tamoxifen-resistant MCF-7 cells. Endocrinology 144: 1032-1044, 2003

25. Bradford MM: A rapid and sensitive method for the quantitation of microgram quantities of protein utilizing the principle of protein-dye binding. Anal Biochem 72: 248-254, 1976.

26. Auerbach R, Kubai L, Knighton D and Folkman J: A simple procedure for the long-term cultivation of chicken embryos. Dev Biol 41: 391-394, 1974.

27. Lee JS, Kang Y, Kim JT, Thapa D, Lee ES and Kim JA: The anti-angiogenic and anti-tumor activity of synthetic phenylpropenone derivatives is mediated through the inhibition of receptor tyrosine kinases. Eur J Pharmacol 677: 22-30, 2012.

28. Religa P, Cao R, Religa D, Xue Y, Bogdanovic N, Westaway D, Marti HH, Winblad B and Cao Y: VEGF significantly restores impaired memory behavior in Alzheimer's mice by improvement of vascular survival. Sci Rep 3: 2053, 2013.

29. Al Zaid Siddiquee K and Turkson J: STAT3 as a target for inducing apoptosis in solid and hematological tumors. Cell Res 18: 254-267, 2008

30. Siveen KS, Sikka S, Surana R, Dai X, Zhang J, Kumar AP, Tan BK, Sethi G and Bishayee A: Targeting the STAT3 signaling pathway in cancer: Role of synthetic and natural inhibitors. Biochim Biophys Acta 1845: 136-154, 2014

31. Marson LP, Kurian KM, Miller WR and Dixon JM: The effect of tamoxifen on breast tumour vascularity. Breast Cancer Res Treat 66: 9-15, 2001.

32. Kongkaneramit L, Sarisuta N, Azad N, Lu Y, Iyer AK, Wang L and Rojanasakul Y: Dependence of reactive oxygen species and FLICE inhibitory protein on lipofectamine-induced apoptosis in human lung epithelial cells. J Pharmacol Exp Ther 325: 969-977, 2008.

33. Yager JD and Davidson NE: Estrogen carcinogenesis in breast cancer. N Engl J Med 354: 270-282, 2006.

34. Garcia-Becerra R, Santos N, Diaz L and Camacho J: Mechanisms of resistance to endocrine therapy in breast cancer: Focus on signaling pathways, miRNAs and genetically based resistance. Int J Mol Sci 14: 108-145, 2012.

35. Goetz MP, Rae JM, Suman VJ, Safgren SL, Ames MM, Visscher DW, Reynolds C, Couch FJ, Lingle WL, Flockhart DA, et al: Pharmacogenetics of tamoxifen biotransformation is associated with clinical outcomes of efficacy and hot flashes. J Clin Oncol 23: 9312-9318, 2005.

36. Hosford SR and Miller TW: Clinical potential of novel therapeutic targets in breast cancer: CDK4/6, Src, JAK/STAT, PARP, HDAC, and PI3K/AKT/mTOR pathways. Pharmgenomics Pers Med 7: 203-215, 2014

37. Smith IE, Walsh G, Skene A, Llombart A, Mayordomo JI, Detre S, Salter J, Clark E, Magill P and Dowsett M: A phase II placebo-controlled trial of neoadjuvant anastrozole alone or with gefitinib in early breast cancer. J Clin Oncol 25: 3816-3822, 2007.

38. Johnston SR, Martin LA, Leary A, Head J and Dowsett M: Clinical strategies for rationale combinations of aromatase inhibitors with novel therapies for breast cancer. J Steroid Biochem Mol Biol 106: 180-186, 2007.

39. Matthes T, Manfroi B and Huard B: Revisiting IL-6 antagonism in multiple myeloma. Crit Rev Oncol Hematol 105: 1-4, 2016.

40. Darnell JE Jr, Kerr IM and Stark GR: Jak-STAT pathways and transcriptional activation in response to IFNs and other extracellular signaling proteins. Science 264: 1415-1421, 1994.

41. Taga T, Hibi M, Hirata Y, Yamasaki K, Yasukawa K, Matsuda T, Hirano $\mathrm{T}$ and Kishimoto T: Interleukin-6 triggers the association of its receptor with a possible signal transducer, gp130. Cell 58: 573-581, 1989.

42. Yu H, Lee H, Herrmann A, Buettner R and Jove R: Revisiting STAT3 signalling in cancer: New and unexpected biological functions. Nat Rev Cancer 14: 736-746, 2014.

43. O'Shea JJ, Schwartz DM, Villarino AV, Gadina M, McInnes IB and Laurence A: The JAK-STAT pathway: Impact on human disease and therapeutic intervention. Annu Rev Med 66: 311-328, 2015

44. Aparicio-Siegmund S, Sommer J, Monhasery N, Schwanbeck R, Keil E, Finkenstädt D, Pfeffer K, Rose-John S, Scheller J and Garbers C: Inhibition of protein kinase II (CK2) prevents induced signal transducer and activator of transcription (STAT) 1/3 and constitutive STAT3 activation. Oncotarget 5: 2131-2148, 2014 
45. Buchert M, Burns CJ and Ernst M: Targeting JAK kinase in solid tumors: Emerging opportunities and challenges. Oncogene 35: 939-951, 2016.

46. Yang S, Luo C, Gu Q, Xu Q, Wang G, Sun H, Qian Z, Tan Y, Qin Y, Shen Y, et al: Activating JAK1 mutation may predict the sensitivity of JAK-STAT inhibition in hepatocellular carcinoma. Oncotarget 7: 5461-5469, 2016.

47. Lee S, Shah T, Yin C, Hochberg J, Ayello J, Morris E, van de Ven $C$ and Cairo MS: Ruxolitinib significantly enhances in vitro apoptosis in Hodgkin lymphoma and primary mediastinal B-cell lymphoma and survival in a lymphoma xenograft murine model. Oncotarget 9: 9776-9788, 2018.

48. Buettner R, Mora LB and Jove R: Activated STAT signaling in human tumors provides novel molecular targets for therapeutic intervention. Clin Cancer Res 8: 945-954, 2002.

49. Bharti AC, Donato $\mathrm{N}$ and Aggarwal BB: Curcumin (diferuloylmethane) inhibits constitutive and IL-6-inducible STAT3 phosphorylation in human multiple myeloma cells. J Immunol 171: 3863-3871, 2003.

50. Stover DG, Gil Del Alcazar CR, Brock J, Guo H, Overmoyer B, Balko J,XuQ,Bardia A, Tolaney SM, Gelman R, et al: Phase II study of ruxolitinib, a selective JAK1/2 inhibitor, in patients with metastatic triple-negative breast cancer. NPJ Breast Cancer 4: 10, 2018.

51. Lee HJ, Seo NJ, Jeong SJ, Park Y, Jung DB, Koh W, Lee HJ Lee EO, Ahn KS, Ahn KS, et al: Oral administration of penta-O-galloyl- $\beta$-D-glucose suppresses triple-negative breast cancer xenograft growth and metastasis in strong association with JAK1-STAT3 inhibition. Carcinogenesis 32: 804-811, 2011.
52. Garg M: Epithelial plasticity and cancer stem cells: Major mechanisms of cancer pathogenesis and therapy resistance. World J Stem Cells 9: 118-126, 2017.

53. Sánchez-Tilló E, Liu Y, de Barrios O, Siles L, Fanlo L, Cuatrecasas M, Darling DS, Dean DC, Castells A and Postigo A: EMT-activating transcription factors in cancer: Beyond EMT and tumor invasiveness. Cell Mol Life Sci 69: 3429-3456, 2012.

54. Smigiel JM, Parameswaran N and Jackson MW: Potent EMT and CSC phenotypes are induced by Oncostatin-M in pancreatic cancer. Mol Cancer Res 15: 478-488, 2017.

55. Wang SW and Sun YM: The IL-6/JAK/STAT3 pathway: Potential therapeutic strategies in treating colorectal cancer (Review). Int J Oncol 44: 1032-1040, 2014.

56. Huang Y, de Reyniès A, de Leval L, Ghazi B, Martin-Garcia N, Travert M, Bosq J, Brière J, Petit B, Thomas E, et al: Gene expression profiling identifies emerging oncogenic pathways operating in extranodal NK/T-cell lymphoma, nasal type. Blood 115: $1226-1237,2010$ 